Łukasz Woźny*

\title{
On the Price of Commitment Assets in a General Equilibrium Model with Credit Constraints and Tempted Consumers
}

DOI 10.1515/bejte-2015-0019

Published online February 3, 2016

Abstract: We analyze a three period production economy, where households exhibit problems of self-control and face credit constraints. Apart from liquid assets, a single commitment (illiquid) asset is available that allows to commit to a planned consumption path. We compare general equilibrium allocations of the two models: one, where households choices are determined using Gul and Pesendorfer (2001, "Temptation and Self-Control." Econometrica 69:1403-35; GP, henceforth) model and the other, where households choices come from a $(\beta-\delta)$ quasi-hyperbolic discounting model. Contrary to the results of Kocherlakota (2001, "Looking for Evidence of Time-Inconsistent Preferences in Asset Market Data." Quarterly Review 13-24) or Gabrieli and Ghosal (2013, "Non-Existence of Competitive Equilibria with Dynamically Inconsistent Preferences." Economic Theory 52:299-313), we show that, when a production sector is incorporated into the economy with commitment asset and credit constraints, we can restore the equilibrium existence (without recalling measure space of consumers (see Luttmer and Mariotti 2006, "Competitive Equilibrium When Preferences Change Over Time." Economic Theory 27:679-90)) and unlike Gul and Pesendorfer (2004b, "Self Control, Revealed Preferences and Consumption Choice." Review of Economic Studies 7:243-64), we show that the equilibrium allocations of both models (GP and $\beta-\delta$ ) imply positive consumption of the commitment asset and corner consumption of one of the liquid assets. We also provide an example showing, when equilibrium allocations of both models are different.

Keywords: time-dependent preferences, time-consistency, self-control, general equilibrium, commitment asset, illiquid bonds, credit constraints

JEL classification: D51, D91

*Corresponding author: tukasz Woźny, Department of Quantitative Economics, Warsaw School of Economics, al. Niepodległości 162, 02-554 Warsaw, Poland, E-mail: lukasz.wozny@sgh.waw.pl 


\section{Introduction}

Since the seminal work of Strotz (1956), there is now an extensive literature stressing the importance of temptation and self-control problems in explaining individual behavior in economic models. When studying dynamic models with such dynamically inconsistent preferences, theoretical economist have developed various solutions methods to explain the behavioral observations that have been found in the empirical literature. ${ }^{1}$ Two predominant frameworks analyzed in the dynamic choice literature include models of the quasi-hyperbolic discounter and the GP model of self-control (see Gul, Pesendorfer (2001)). Economists attempted to analyze and compare various implications of both frameworks in specific applications including: characterizing consumption dynamics of consumers facings temptations (see Laibson (1997) or Balbus, Reffett, and Woźny (2015) for the $\beta-\delta$ model and Gul and Pesendorfer (2004a) for the GP representation), temptation implications on the optimal taxation (see Krusell, Kuruşçu, and Smith (2002) for the $\beta-\delta$ model or Krusell, Kuruşçu, and Smith (2010) for the GP framework), or the optimal incentive schemes for tempted agents (Yilmaz (2013) for the $\beta-\delta$ model and Woźny (2015) for the GP representation) among many others.

Surprisingly, only few papers analyzed temptation/self control implications on equilibrium prices, demand/supply of commitment assets, left alone the existence and properties of a general equilibrium at the theoretical level. Few notable exceptions include Herings and Rohde (2006), Luttmer and Mariotti (2006, 2007), Gabrieli and Ghosal (2013), Dziewulski (2015) or examples presented in Kocherlakota (2001) and Gul and Pesendorfer (2004b). These authors discuss problems with equilibrium existence, characterize competitive equilibrium allocations or present some examples of equilibria, when households exhibit self control problems or have time inconsistent preferences. Importantly to say, all of the above mentioned papers concentrate on the exchange economies or power utilities (see Luttmer and Mariotti 2003).

Specifically and importantly to our research, Gabrieli and Ghosal (2013) showed that competitive equilibrium may not exists in a three period exchange economy (without commitment assets) with a single $\beta-\delta$ (time-inconsistent) consumer. This resulted from (generic) non-monotonicity and non-convexity of the sophisticated consumer's date 1 (implied) preferences (i. e. ones obtained by incorporating date 2 and 3 optimal decisions). Demand calculated in their example do not vary with prices sufficiently to cover the part of domain with

1 See the work of Angeletos, Laibson, Repetto, Tobacman, and Weinberg (2001) or Ameriks, Caplin, Leahy, and Tyler (2007) for empirical motivation. 
fixed supply. Next Kocherlakota (2001) showed ${ }^{2}$ that in the same economy, but with commitment asset and credit constraints, the demand for one of the assets: (liquid) savings in the final date and (illiquid) date-1 commitment asset must be zero. Intuitively, if the commitment asset is relatively cheep, the first period self must leave the second period self which such level of capital (to finance date 2 consumption) that no further savings in date 2 is necessary. If on the other hand, the price of the commitment asset is too high, then the investment in the commitment asset is zero and all consumption in date 3 is covered from savings in date 2. These result from the fact that, if the second period self is unconstrained, than any replacement between assets, such that the consumption in date 2 and 3 is unchanged, leaves the cost of self control (lost efficiency from the date 1 perspective) unchanged, but may reduce costs. The corner demand result of Kocherlakota (2001) causes, however, problems with respect to existence of equilibrium of the exchange economy. Similarly to the result of Gabrieli and Ghosal (2013) the date 1 implied preferences, although concave, are not strictly monotone. In fact, unless the prices are such that consumers are indifferent between investing in the short term or commitment asset, and the number of consumers is large, the demand will not equate supply and equilibrium will not exists. Following the technical argument of Rogerson (1988), he argued that in order for the competitive equilibrium to exists the (large) group of agents must divide into those investing in the commitment asset only and those investing in the liquid assets only. In such case asymmetric equilibrium may exist but as he argued, such equilibrium allocation is not supported by empirical observations. ${ }^{3}$ Importantly, note that the nonexistence example of Gabrieli and Ghosal (2013) cannot be remedied using the large economy framework as the market clearing prices do not belong to the convex hull of demand.

Finally, Gul and Pesendorfer (2004b) (Section 5.1) provided an example showing that, if we model consumer's choice using GP framework, in the competitive equilibrium of the exchange economy, we may observe agents possessing strictly positive demands of both assets, i. e. commitment and short term bonds. The intuition used to understand the result in the $\beta-\delta$ model is not longer useful here. Even if the commitment asset is expensive, it may be used to cover some, but not all, date 3 consumption. On the contrary to the $\beta-\delta$ model, in the GP framework any change in assets such that date 2 and date 3 consumption is unchanged, changes the cost of self-control, as it depends on the level of

2 See also Laibson (1997) for a similar observation.

3 See also Luttmer and Mariotti (2006) for a related argument for existence of a competitive equilibrium with time-inconsistent preferences and a measure space of agents, but no commitment assets. 
date 2 liquid but not illiquid asset. Finally to mention, GP model does also allow for the corner solution, if prices are appropriate. As a result, Gul and Pesendorfer (2004b) claimed that GP representation/model possesses an advantage over the $\beta-\delta$ one, as it can model non-corner choices of all three assets and, as a result, a (symmetric) equilibrium in the exchange economy with finite number of consumers may exist.

The aim of this paper is to extend the analysis of Kocherlakota (2001) (or Gabrieli and Ghosal (2013)) and Gul and Pesendorfer (2004b) (Section 5.1) to a three period production economy with commitment assets and credit constraints, and verify, if the results of both, the above mentioned models, remain discrepant. Specifically, we prove the existence of equilibrium in both models and study how the equilibrium allocations and prices differ between both models.

From this perspective the contribution of this paper is threefold. First, we argue that, when the production side of the economy is incorporated into both of the above mentioned models (with commitment (illiquid) asset and credit constraints), the equilibrium allocation is characterized by the positive consumption of the commitment asset and corner consumption of one of the short term assets. Contrary to the examples for the exchange economy, the results of both threeperiod models with production are hence similar. Still, there are differences between both models, especially seen if we consider more than three periods. Second, in the paper we provide examples, when solutions of both models are indeed discrepant. Three and finally, we prove the equilibrium existence in the three-period production economy of a representative agent. The reason we restore equilibrium existence in our model is the joint presence of commitment assets together with credit constraints and active firms. In fact, the corner allocation results we obtain is necessary to avoid problems mentioned by Gabrieli and Ghosal (2013) and presence of the firm helps to avoid problems identified by Kocherlakota (2001), i.e. allow demand/supply to vary sufficiently with prices to clear the market.

In the rest of the paper, we first study the firm's (Section 2) and then the consumer's maximization (for GP and $\beta-\delta$ ) (Section 3) and summarize our results with equilibrium analysis (Section 4). Section 5 discusses few extensions of our basic model by allowing for more (liquid) assets, and more than three periods.

\section{Firm's Behavior}

Consider a firm possessing technology given by a production function $F$, defined over capital and labor inputs. We assume $F: \mathbb{R}_{+} \times \mathbb{R}_{+} \rightarrow \mathbb{R}_{+}$has constant 
returns to scale, is weekly concave, is strictly monotone and strictly concave with each argument separately for a positive input of the other argument, twice continuously differentiable and satisfies Inada conditions. Moreover, both inputs are required for production: $F(k, 0)=0$ and $F(0, l)=0$. Firm has no endowment of capital. Each date firm hires labor $l_{t}$ at wage $w_{t} \in \mathbb{R}_{++}$as well as rents capital $k_{t} \geq 0$ and returns $1+r_{t}$ at the end of the period. In date 1 firm can also rent a (long term) capital ${ }^{4} a$. Once deciding to do so, $a$ is used in date 2 and 3 and is returned with interest $r_{a}$ at the end of date $3 .^{5}$ There is no capital depreciation and $r_{t}, r_{a} \in \mathbb{R}_{++}$Firm discounts its profits using $q_{t}=\frac{1}{1+r_{t+1}}$. Price $p$ of (date 1) consumption good is normalized to 1 . A problem of the firm is then to solve:

$$
\begin{array}{rl}
\max _{k_{1}, k_{2}, k_{3}, l_{1}, l_{2}, l_{3}, a \geq 0} & F\left(k_{1}, l_{1}\right)-w_{1} l_{1}-r_{1} k_{1}+ \\
& q_{1}\left[F\left(a+k_{2}, l_{2}\right)-w_{2} l_{2}-r_{2} k_{2}\right]+ \\
q_{1} q_{2}\left[F\left(a+k_{3}, l_{3}\right)+a-w_{3} l_{3}-r_{3} k_{3}-\left(1+r_{a}\right) a\right] .
\end{array}
$$

As asset returns are finite the Inada conditions assumed on $F$ imply that $l_{1}, l_{2}, l_{3}$ and $k_{1}$ are interior. However, as a and $k_{2}$ (and $k_{3}$ ) are substitutes it is not clear is all of these are interior. Knowing that we obtain the first order conditions:

$$
\begin{aligned}
& F_{2}^{\prime}\left(k_{1}, l_{1}\right)=w_{1}, F_{2}^{\prime}\left(a+k_{2}, l_{2}\right)=w_{2}, F_{2}^{\prime}\left(a+k_{3}, l_{3}\right)=w_{3}, F_{1}^{\prime}\left(k_{1}, l_{1}\right)=r_{1}, \\
& F_{1}^{\prime}\left(a+k_{2}, l_{2}\right)=r_{2}-\mu_{2}, F_{1}^{\prime}\left(a+k_{3}, l_{3}\right)=r_{3}-\mu_{3}, \\
& F^{\prime}{ }_{1}\left(a+k_{2}, l_{2}\right)+\mu_{a}+q_{2}\left[F_{1}^{\prime}\left(a+k_{3}, l_{3}\right)+1-\left(1+r_{a}\right)\right]=0,
\end{aligned}
$$

where $\left(\mu_{i}\right)_{i=1,2}$ are Lagrange multipliers associated with non-negativity constraints: $-k_{i} \leq 0$ and $\mu_{a}$ with $-a \leq 0$. We start with the following lemmas:

Lemma 1: Suppose $\left(1+r_{2}\right)\left(1+r_{3}\right)<1+r_{a}$, then the optimal $a=0$ and $k_{2} k_{3}>0$.

Proof: Suppose not, i. e. a $>0$. Then, consider a new choice $a^{\prime}=a-\epsilon, k_{3}^{\prime}=k_{3}+\epsilon$, $k_{2}^{\prime}=k_{2}+\epsilon$ for some positive $\epsilon<a$. Observe that this leaves the production in both

4 Observe that unlike Kocherlakota (2001) or Gul and Pesendorfer (2004b) we do not consider a long term, liquid asset but analyze, instead, two short term bonds $k_{2}, k_{3}$. This is actually equivalent but allows to model firm's choices in an easier way. Indeed, the composition of bonds in date 2 and 3 can be treated as the long term (liquid) asset with return $\left(1+r_{2}\right)\left(1+r_{3}\right)$. See section 5 for a formal argument.

5 Here we assume that both the long- and the short-term capitals are perfect substitutes in the production process in the second and the third period. The reason, why a is not used in period 1 is justified, as there is no initial endowment of the commitment asset and the consumption and saving choices of the consumer are determined at the end of each period after production took place. 
periods unchanged. Allocation prime costs less, however. To see that, observe that such $\epsilon$-change affects cost the following way:

$$
\begin{aligned}
& r_{2}\left(k_{2}^{\prime}-k_{2}\right)+q_{2}\left[r_{a}\left(a^{\prime}-a\right)+r_{3}\left(k_{3}^{\prime}-k_{3}\right)\right]=\frac{\epsilon}{q_{2}}\left[r_{2}\left(1+r_{3}\right)+r_{3}-r_{a}\right]= \\
& \frac{\epsilon}{q_{2}}\left[r_{2}\left(1+r_{3}\right)+\left(1+r_{3}\right)-\left(1+r_{a}\right)\right]=\frac{\epsilon}{q_{2}}\left[\left(1+r_{2}\right)\left(1+r_{3}\right)-\left(1+r_{a}\right)\right]<0 .
\end{aligned}
$$

Profit increases after such change, hence the original allocation could not be optimal.

Lemma 2: Suppose $\left(1+r_{2}\right)\left(1+r_{3}\right)>1+r_{a}$, then, $a>0$ and $k_{2} k_{3}=0$ at the optimal choice.

Proof: Suppose the optimal $k_{2}$ and $k_{3}$ are positive. Consider an allocation: $a^{\prime}=a+\epsilon, k_{3}^{\prime}=k_{3}-\epsilon, k_{2}^{\prime}=k_{2}-\epsilon$ for some positive $\epsilon<\min \left\{k_{2}, k_{3}\right\}$. Such allocation leaves production in both periods unchanged. Observe change in costs:

$$
\begin{aligned}
& r_{2}\left(k_{2}^{\prime}-k_{2}\right)+q_{2}\left[r_{a}\left(a^{\prime}-a\right)+r_{3}\left(k_{3}^{\prime}-k_{3}\right)\right]=-\frac{\epsilon}{q_{2}}\left[r_{2}\left(1+r_{3}\right)+r_{3}-r_{a}\right]= \\
& -\frac{\epsilon}{q_{2}}\left[r_{2}\left(1+r_{3}\right)+\left(1+r_{3}\right)-\left(1+r_{a}\right)\right]=-\frac{\epsilon}{q_{2}}\left[\left(1+r_{2}\right)\left(1+r_{3}\right)-\left(1+r_{a}\right)\right]<0 .
\end{aligned}
$$

Again cost is reduced and hence profit increases. This contradicts optimality of the original allocation.

Both lemmas characterize the demand of liquid and illiquid assets for given factor prices. For the case of $\left(1+r_{a}\right)=\left(1+r_{2}\right)\left(1+r_{3}\right)$ the solution exists but it not unique.

We now shortly comment that the use of commitment asset shifts profits between the periods. So, denote by $\pi_{t}$ date $\mathrm{t}$ optimal profits, i. e. $\pi_{1}=F\left(k_{1}, l_{1}\right)-$ $w_{1} l_{1}-r_{1} k_{1}, \quad \pi_{2}=F\left(a+k_{2}, l_{2}\right)-w_{2} l_{2}-r_{2} k_{2}$ and $\pi_{3}=F\left(a+k_{3}, l_{3}\right)-w_{3} l_{3}-r_{3} k_{3}-r_{a}$. Clearly $\pi_{1}=0$, but for a positive demand of illiquid assets, profit is shifted from $\pi_{3}$ to $\pi_{2}$. The next lemma formalizes this result.

Lemma 3: Suppose the optimal $k_{2}>0$, then $\pi_{2} \geq 0$, with strict inequality if $a>0$. Similarly, if the optimal $k_{3}>0$, then $\pi_{3} \leq 0$ with strict inequality if $a>0$. Finally, if the optimal $a=0$, then $\pi_{2}=\pi_{3}=0$.

\section{Proof:}

$$
\begin{aligned}
\pi_{2} & =F\left(a+k_{2}, l_{2}\right)-w_{2} l_{2}-r_{2} k_{2}=\left(a+k_{2}\right) F_{1}^{\prime}\left(a+k_{2}, l_{2}\right)+l_{2} F^{\prime}{ }_{2}\left(a+k_{2}, l_{2}\right)-w_{2} l_{2}-r_{2} k_{2}= \\
& =a F^{\prime}{ }_{1}\left(a+k_{2}, l_{2}\right) \geq 0 .
\end{aligned}
$$


Similarly:

$$
\begin{aligned}
\pi_{3} & =F\left(a+k_{3}, l_{3}\right)-w_{3} l_{3}-r_{3} k_{3}-r_{a} a= \\
& =\left(a+k_{3}\right) F_{1}^{\prime}\left(a+k_{3}, l_{3}\right)+l_{3} F_{2}^{\prime}\left(a+k_{3}, l_{3}\right)-w_{3} l_{3}-r_{3} k_{3}-r_{a} a \\
& \leq a\left(F^{\prime}{ }_{1}\left(a+k_{3}, l_{3}\right)-r_{a}\right) \leq 0,
\end{aligned}
$$

where the last inequality follows from the first order conditions for the optimal $a$ :

$$
r_{a}=F^{\prime}{ }_{1}\left(a+k_{3}, l_{3}\right)+\frac{\mu_{a}+F_{1}^{\prime}\left(a+k_{2}, l_{2}\right)}{q_{2}}>F^{\prime}{ }_{1}\left(a+k_{3}, l_{3}\right) .
$$

Finally if $a=0$, then zero profit follows from constant returns to scale assumption.

\section{Consumer's Behavior}

We now turn to characterize the consumer's side of the market, starting with the GP preferences and then moving to the $\beta-\delta$ ones. Consumer is endowed with initial capital $k_{1}>0$ and one unit of free time each period. As we assume no disutility from labour, its supply is fixed and $l_{t}=1$ in both models.

\subsection{GP Consumer's Behavior}

Consider a household with the GP type preferences defined over consumption in three periods:

$$
\sum_{t=1}^{3} \beta^{t-1}\left\{u\left(c_{t}\right)+v\left(c_{t}\right)-v\left(m_{t}\right)\right\}
$$

where $u: \mathbb{R}_{+} \rightarrow \mathbb{R}$ stands for commitment utility and $v: \mathbb{R}_{+} \rightarrow \mathbb{R}$ for temptation utility and $m_{\mathrm{t}}$ is the value of date $t$ liquid assets. Both utilities are strictly increasing, strictly concave and twice continuously differentiable and satisfy Inada conditions. Here we assume that the temptation calls for consuming the whole liquid asset. Initial capital $k_{1}>0$ is given. Consumer invests its income in four assets $k_{1}$, $k_{2}, k_{3}, a$. Assets $k_{2}$ and $k_{3}$ play a role of short (one-period) bonds, while $a$ a longterm (illiquid) commitment asset. As we assume that the consumer is not endowed with the commitment asset, as opposed to $k_{1}$, it cannot be supplied and used in the production process in period 1 . Asset holdings cannot be negative. ${ }^{6}$ Consumer also works earning $w_{\mathrm{t}}$ each date and owns a firm providing profits $\pi_{1}, \pi_{2}, \pi_{3}$.

6 See discussion in Laibson (1997) page 448 for arguments, why it is so. 
Formally the GP consumer maximization problem is given by:

$$
\begin{aligned}
\max _{c_{1}, c_{2}, c_{3}, k_{2}, k_{3}, a \geq 0} & \sum_{t=1}^{3} \beta^{t-1}\left\{u\left(c_{t}\right)+v\left(c_{t}\right)-v\left(m_{t}\right)\right\}, \\
& c_{1}+k_{2}+a=w_{1}+\pi_{1}+k_{1}\left(1+r_{1}\right)=: m_{1}, \\
\text { s.t. } & c_{2}+k_{3}=w_{2}+\pi_{2}+k_{2}\left(1+r_{2}\right)=: m_{2}, \\
& c_{3}=w_{3}+\pi_{3}+k_{3}\left(1+r_{3}\right)+a\left(1+r_{a}\right)=: m_{3} .
\end{aligned}
$$

Clearly, supply of $k_{1}$ is fixed for any $r_{1} \geq 0$ and nontrivial choice of only three assets must be considered. Also observe that as $m_{1}$ is given, although the term $v\left(m_{1}\right)=v\left(w_{1}+\pi_{1}+\left(1+r_{1}\right) k_{1}\right)$ changes the optimal value it does not influence the optimal choice. Also, in the terminal date household consumes the whole available in date 3 liquid asset, hence $u\left(c_{3}\right)+v\left(c_{3}\right)-v\left(m_{3}\right)=u\left(m_{3}\right)+$ $v\left(m_{3}\right)-v\left(m_{3}\right)=u\left(m_{3}\right)$. As a result, the consumer problem reduces to:

$$
\begin{aligned}
\max _{k_{2}, k_{3}, a} & \sum_{t=1}^{2} \beta^{t-1}\left\{u\left(c_{t}\right)+v\left(c_{t}\right)\right\}+\beta^{2} u\left(c_{3}\right)-\beta v\left(w_{2}+\pi_{2}+k_{2}\left(1+r_{2}\right)\right), \\
\text { s.t. } & c_{1}+k_{2}+a=w_{1}+\pi_{1}+k_{1}\left(1+r_{1}\right), \\
& c_{2}+k_{3}=w_{2}+\pi_{2}+k_{2}\left(1+r_{2}\right), \\
& c_{3}=w_{3}+\pi_{3}+k_{3}\left(1+r_{3}\right)+a\left(1+r_{a}\right), \\
& -k_{2} \leq 0, \quad-k_{3} \leq 0, \quad-a \leq 0 .
\end{aligned}
$$

Note, here we have dropped the constraints implying nonnegativity of consumption. This is without loss of generality, as by Inada conditions on $u$, optimal $c_{t}>0$. Associate Lagrange multipliers $\lambda_{t}$ with each inequality constraint and consider the first order conditions for the optimal portfolio:

$$
\begin{aligned}
u^{\prime}\left(c_{1}\right)+v^{\prime}\left(c_{1}\right) & =\beta\left(1+r_{2}\right)\left[u^{\prime}\left(c_{2}\right)+v^{\prime}\left(c_{2}\right)-v^{\prime}\left(w_{2}+\pi_{2}+k_{2}\left(1+r_{2}\right)\right)\right]+\lambda_{2}, \\
u^{\prime}\left(c_{1}\right)+v^{\prime}\left(c_{1}\right) & =\beta^{2}\left(1+r_{a}\right) u^{\prime}\left(c_{3}\right)+\lambda_{a}, \\
\beta\left(u^{\prime}\left(c_{2}\right)+u^{\prime}\left(c_{2}\right)\right) & =\beta^{2}\left(1+r_{3}\right) u^{\prime}\left(c_{3}\right)+\lambda_{3} .
\end{aligned}
$$

By rearranging we obtain:

$$
\beta^{2} u^{\prime}\left(c_{3}\right)\left[\left(1+r_{a}\right)-\left(1+r_{2}\right)\left(1+r_{3}\right)\right]=\beta\left(1+r_{2}\right)\left[\frac{\lambda_{3}}{\beta}-v^{\prime}\left(w_{2}+\pi_{2}+k_{2}\left(1+r_{2}\right)\right)\right]+\lambda_{2}-\lambda_{a} \text {. }
$$

Using this condition we characterize the supply for capital and commitment asset $a$.

Lemma 4: Suppose $\left(1+r_{a}\right) \geq\left(1+r_{2}\right)\left(1+r_{3}\right)$, then optimal $k_{2} k_{3}=0$.

Proof: The observation follows directly from the necessary condition (4), requiring that $\lambda_{2}>0$ or $\lambda_{3}>0$. 
In the case of $\left(1+r_{a}\right)<\left(1+r_{2}\right)\left(1+r_{3}\right)$, positive supply of all three assets is possible.

\section{$3.2 \beta-\delta$ Consumer's Behavior}

Consider the $\beta$ - $\delta$ preferences over the three periods: $u\left(c_{1}\right)+\beta \delta\left(u\left(c_{2}\right)+\delta u\left(c_{3}\right)\right)$, where after choosing $c_{1}$ the choice of $c_{2}$ and $c_{3}$ is determined by maximizing utility $u\left(c_{2}\right)+\beta \delta u\left(c_{3}\right)$. That is, we consider the sophisticated consumer or time consistent solution to the consumer's problem. We consider the same set of assets and constraints as in the previous subsection. Formally, the $\beta$ - $\delta$ consumer's maximization problem is given by:

$$
\begin{array}{cl}
\max _{c_{1}, k_{2}, a \geq 0} & u\left(c_{1}\right)+\beta \delta\left(u\left(c_{2}^{\star}\left(k_{2}, a\right)\right)+\delta u\left(c_{3}^{\star}\left(k_{2}, a\right)\right)\right) \\
\text { s. t. } & c_{1}+k_{2}+a=w_{1}+\pi_{1}+k_{1}\left(1+r_{1}\right),
\end{array}
$$

where $c_{2}^{\star}\left(k_{2}, a\right)$ and $c_{3}^{\star}\left(k_{2}, a\right)$ are such that:

$$
\begin{aligned}
& \left(c_{2}^{\star}\left(k_{2}, a\right), c_{3}^{\star}\left(k_{2}, a\right), k_{3}^{\star}\left(k_{2}, a\right)\right) \in \arg \max _{c_{2}, c_{3}, k_{3} \geq 0} u\left(c_{2}\right)+\beta \delta u\left(c_{3}\right) \\
& \text { s.t. } \quad c_{2}+k_{3}=w_{2}+\pi_{2}+k_{2}\left(1+r_{2}\right) \text {, } \\
& c_{3}=w_{3}+\pi_{3}+k_{3}\left(1+r_{3}\right)+a\left(1+r_{a}\right) \text {, }
\end{aligned}
$$

We denote the first maximization as the self 1 problem and decision maker in the second problem as self 2 . Let $\beta \in(0,1), \delta \in(0,1]$ and, as previously, assume that $u: \mathbb{R}_{+} \rightarrow \mathbb{R}$ is strictly increasing, strictly concave and twice continuously differentiable with Inada conditions satisfied. We start with a simple lemma:

Lemma 5: Suppose $\left(1+r_{a}\right)<\left(1+r_{2}\right)\left(1+r_{3}\right)$, then $a k_{3}=0$ at the optimal choice.

Proof: Suppose not, i. e. both $a>0$ and $k_{3}>0$. Consider a new decision of self 1 $a^{\prime}=a-\epsilon$ and $k_{2}^{\prime}=k_{2}+\epsilon \frac{1+r_{a}}{\left(1+r_{2}\right)\left(1+r_{3}\right)}$. Under such allocation self 2 would choose the same level of consumption as before by allocating $k_{3}^{\prime}=k_{3}+\epsilon \frac{1+r_{a}}{1+r_{3}}$. If self 2 was unconstrained before, he is still unconstrained and can afford $c_{2}, c_{3}$, hence: $c^{\prime}{ }_{2}=c_{2}$ and $c^{\prime}{ }_{3}=c_{3}$. Such change, however, makes consumer 1 wealthier. To see that, follow the change in costs:

$$
-\epsilon+\epsilon \frac{1+r_{a}}{\left(1+r_{2}\right)\left(1+r_{3}\right)}=\epsilon\left[-1+\frac{1+r_{a}}{\left(1+r_{2}\right)\left(1+r_{3}\right)}\right]<0 .
$$

Lemma 6: Suppose $\left(1+r_{a}\right) \geq\left(1+r_{2}\right)\left(1+r_{3}\right)$, then optimal $k_{3}=0$. 
Proof: Suppose not, i. e. $k_{3}>0$. By $c_{1}^{\star}, c_{2}^{\star}, c_{3}^{\star}$ denote the optimal consumption plan with such $k_{t}$. As $k_{3}>0$ we know that $u^{\prime}\left(c_{2}^{*}\right)=\left(1+r_{3}\right) \beta \delta u^{\prime}\left(c_{3}^{*}\right)$. By $c_{2}^{\prime}, c_{3}^{\prime}$ denote solution to the following maximization problem:

$$
\begin{aligned}
& \max _{c_{2}, c_{3} \geq 0} u\left(c_{2}\right)+\delta u\left(c_{3}\right), \\
& \text { s. t. } c_{2}+\frac{c_{3}}{1+r_{3}} \leq c_{2}^{\star}+\frac{c_{3}^{*}}{1+r_{3}}, \\
& \text { and } c_{2} \geq \pi_{2}+w_{2} .
\end{aligned}
$$

We obtain the first order condition $u^{\prime}\left(c_{2}^{\prime}\right)=\left(1+r_{3}\right) \delta u^{\prime}\left(c^{\prime}{ }_{3}\right)+\mu$. We also know that $\mu+\left(1+r_{3}\right) \delta u^{\prime}\left(c_{3}^{\prime}\right)>\left(1+r_{3}\right) \beta \delta u^{\prime}\left(c_{3}^{\prime}\right)$ hence $c_{3}^{\prime}>c_{3}^{*}$ and $c_{2}^{\prime}>c_{2}^{*}$. This implies that $c_{2}^{\star}>\pi_{2}+w_{2}$. As a result pair $c_{2}^{\star}, c_{3}^{\star}$ satisfies constraints, but is different from the optimal one, hence:

$$
u\left(c_{2}^{\prime}\right)+\delta u\left(c_{3}^{\prime}\right)>u\left(c_{2}^{\star}\right)+\delta u\left(c_{3}^{\star}\right)
$$

Now let $a^{\prime}=\frac{c_{3}^{\prime}-w_{3}-\pi_{3}}{1+r_{a}}$ and $k_{2}^{\prime}=\frac{c_{2}^{\prime}-w_{2}-\pi_{2}}{1+r_{2}}$. By construction $k_{2}^{\prime} \geq 0$ and $a^{\prime}>a \geq 0$. Observe that period 2 self faced with such endowment chooses $k_{3}^{\prime}=0$ as:

$$
\frac{u^{\prime}\left(c_{2}^{\prime}\right)}{\beta \delta u^{\prime}\left(c_{2}^{\prime}\right)}>1+r_{3}=\frac{u^{\prime}\left(c_{2}^{\prime}\right)}{\delta u^{\prime}\left(c_{2}^{\prime}\right)},
$$

and he is constrained with $k_{3} \geq 0$. As a result, for $a^{\prime}, k^{\prime}{ }_{2}$ period 2 self chooses $c^{\prime}{ }_{2}$, $c_{3}^{\prime}$ which gives higher utility to period one self by inequality [6].

We now claim that period 1 self can afford choice of $c_{1}^{\star}, a^{\prime}, k_{2}^{\prime}$. To see that follow:

$$
\begin{aligned}
& c_{1}^{\star}+k_{2}^{\prime}+a^{\prime}=c_{1}^{\star}+\frac{c_{2}^{\prime}-w_{2}-\pi_{2}}{1+r_{2}}+\frac{c_{3}^{\prime}-w_{3}-\pi_{3}}{1+r_{a}}= \\
& c_{1}^{\star}+\frac{c_{2}^{\prime}-w_{2}-\pi_{2}}{1+r_{2}}+\frac{c_{3}^{\prime}-w_{3}-\pi_{3}}{\left(1+r_{2}\right)\left(1+r_{3}\right)}+\left(c_{3}^{\prime}-w_{3}-\pi_{3}\right)\left[\frac{1}{1+r_{a}}-\frac{1}{\left(1+r_{2}\right)\left(1+r_{3}\right)}\right]= \\
& c_{1}^{\star}+\frac{c_{2}^{\star}-w_{2}-\pi_{2}}{1+r_{2}}+\frac{c_{3}^{\star}-w_{3}-\pi_{3}}{\left(1+r_{2}\right)\left(1+r_{3}\right)}+\left(c_{3}^{\prime}-w_{3}-\pi_{3}\right)\left[\frac{1}{1+r_{a}}-\frac{1}{\left(1+r_{2}\right)\left(1+r_{3}\right)}\right]= \\
& c_{1}^{\star}+k_{2}+\frac{a\left(1+r_{a}\right)}{\left(1+r_{2}\right)\left(1+r_{3}\right)}+\left(c_{3}^{\prime}-w_{3}-\pi_{3}\right)\left[\frac{1}{1+r_{a}}-\frac{1}{\left(1+r_{2}\right)\left(1+r_{3}\right)}\right]= \\
& c_{1}^{\star}+k_{2}+a+\left(1+r_{a}\right)\left(a^{\prime}-a\right)\left[\frac{1}{1+r_{a}}-\frac{1}{\left(1+r_{2}\right)\left(1+r_{3}\right)}\right] \leq c_{1}^{\star}+k_{2}+a .
\end{aligned}
$$

This yields a contradiction. We have found an affordable, time-consistent allocation that gives higher utility to period 1 self. 


\section{Competitive Equilibrium}

We now turn to compare the equilibrium implications for both representations of temptation preferences: GP and $\beta-\delta$ one. We define:

Definition 1: A competitive equilibrium with GP (or $\beta-\delta$ respectively) consumer is a list of non-negative quantities $\left\{c_{t}\right\}_{t=1}^{3},\left\{k_{t}\right\}_{t=1}^{3}, a,\left\{l_{t}\right\}_{t=1}^{3}, a^{f},\left\{l_{t}^{f}\right\}_{t=1}^{3}$, profits $\left\{\pi_{t}\right\}_{t=1}^{3}$, and non-negative prices $\left\{r_{t}\right\}_{t=1}^{3}, r_{a},\left\{w_{t}\right\}_{t=1}^{3}$ such that:

1. taking prices $\left\{r_{t}\right\}_{t=1}^{3}, \mathrm{r}_{\mathrm{a}},\left\{w_{t}\right\}_{t=1}^{3}$ as given, quantities $\left\{k_{t}^{f}, l_{t}^{f}\right\}_{t=1}^{3}, \mathrm{a}^{\mathrm{f}}$ solve the firm's maximization problem given by problem (1) and $\left\{\pi_{t}\right\}_{t=1}^{3}$ are the firm's profits,

2. taking prices $\left\{r_{t}\right\}_{t=1}^{3}, \mathrm{r}_{\mathrm{a}},\left\{w_{t}\right\}_{t=1}^{3}$ and profits $\left\{\pi_{t}\right\}_{t=1}^{3}$ as given, quantities $\left\{c_{t}\right\}_{t=1}^{3},\left\{k_{t}\right\}_{t=2}^{3}$, a, solve the consumer maximization problem given by problem (3) (or (5) respectively) and $\mathrm{l}_{t}=1$ for $\mathrm{t}=1,2,3$ with $\mathrm{k}_{1}$ given,

3. markets clear, i.e.: for $\mathrm{t}=1,2,3$ we have $k_{t}=k_{t}^{f}, l_{t}=l_{t}^{f}, a=a^{f}$, as well as $c_{1}+a+k_{2}=F\left(k_{1}^{f}, l_{1}^{f}\right)+k_{1}, c_{2}+k_{3}=F\left(a^{f}+k_{2}^{f}, l_{2}^{f}\right)+k_{2}$ and $c_{3}=F\left(a^{f}+k_{3}^{f}, f_{3}^{f}\right)+$ $k_{3}+a$.

Hence, by a competitive equilibrium we mean a list of quantities (solving both the consumer and the producer problems) and prices such that markets for all assets clear.

We start by providing few results characterising the equilibrium allocations.

Proposition 1: For both types of preferences (GP and $\beta-\delta$ ), in any competitive equilibrium $\left(1+r_{a}\right) \leq\left(1+r_{2}\right)\left(1+r_{3}\right)$.

Proof: We start with the GP case. Suppose the opposite, i.e. $\left(1+r_{a}\right)>$ $\left(1+r_{2}\right)\left(1+r_{3}\right)$. Then, lemma 1 implies $k_{2} k_{3}>0$, while lemma $4 k_{2} k_{3}=0$. This taken together contradicts market clearing. Similarly for a $\beta-\delta$ consumer. Suppose that $\left(1+r_{a}\right)>\left(1+r_{2}\right)\left(1+r_{3}\right)$. Then, lemma $1\left(k_{2} k_{3}>0\right)$ together with lemma 6 (implying $k_{3}=0$ ) contradicts market clearing.

Proposition 2: For GP consumer, in any competitive equilibrium we have $k_{2} k_{3}=0$, while for the $\beta-\delta$ consumer, we have $k_{3}=0$.

Proof: For GP consumer, by proposition 1 we know that in equilibrium $\left(1+r_{a}\right) \leq\left(1+r_{2}\right)\left(1+r_{3}\right)$. We have two cases: for $\left(1+r_{a}\right)=\left(1+r_{2}\right)\left(1+r_{3}\right)$ the thesis follows from lemma 4 , while for $\left(1+r_{a}\right)<\left(1+r_{2}\right)\left(1+r_{3}\right)$ the thesis follows from lemma 2. 
For $\beta-\delta$ consumer, if $\left(1+r_{a}\right)=\left(1+r_{2}\right)\left(1+r_{3}\right)$, then the thesis is clear from lemma 6 . Also for $\left(1+r_{a}\right)<\left(1+r_{2}\right)\left(1+r_{3}\right)$ the thesis is clear by lemma 2 and 5 , as otherwise we would obtain a contradiction.

This implies that in equilibrium we cannot observe positive allocation of all three assets, i. e. $a k_{2} k_{3}=0$.

Proposition 3: For both GP and $\beta-\delta$ consumer in any competitive equilibrium we have $a>0$.

Proof: We start with GP consumer. By proposition 2 we know that in any equilibrium $k_{2} k_{3}=0$.

If in equilibrium $k_{2}=0$ and $a=0$, then $\pi_{2}+w_{2}=0=c_{2}$. For $r_{2}>0$ the consumer can increase $k_{2}$ by $\varepsilon$, get positive income and afford positive $c_{2}$. He is willing to do so by Inada conditions. Otherwise consumer's maximization is violated. The other case of $r_{2}=0$ cannot be an equilibrium price, markets cannot clear as firm requires infinite demand of $a$.

If in equilibrium $k_{3}=0$ and $a=0$, then $\pi_{3}+w_{3}=0=c_{3}$. Similarly as above consider $r_{3}>0$, but in such a case the consumer can increase $k_{3}$ by $\varepsilon$, get positive income and afford positive $c_{3}$. He is willing to do so by Inada conditions and thus violates consumer's maximization.

For $\beta-\delta$ consumer the thesis is clear for $\left(1+r_{a}\right)<\left(1+r_{2}\right)\left(1+r_{3}\right)$ from lemma 2. For $\left(1+r_{a}\right)=\left(1+r_{2}\right)\left(1+r_{3}\right)$ suppose the contrary, i.e. $a=0$. But this implies that $\pi_{3}+w_{3}=0$. As above for $r_{a}>0$, by Inada condition, consumer wants to transfer some amount to date 3, contradicting $a=0$.

Let us stress, that the existence of competitive equilibrium is not obvious here. Firstly, both types of consumers ${ }^{7}$ have utility functions that are not strictly monotone nor concave. This properties can imply equilibrium nonexistence (see Gabrieli and Ghosal (2013) or Kocherlakota (2001)). For these reasons, we now provide a proof, when the competitive equilibrium indeed exists.

Proposition 4: There exist a competitive equilibrium (for both GP and $\beta-\delta$ consumers) with strictly positive prices.

Proof: Consider the $\beta-\delta$ economy. To simplify notation set $f(k):=F(k, 1)$ and observe $f^{\prime}(k):=F_{1}^{\prime}(k, 1)$. Observe that by proposition 3 there is no equilibrium for $\left(1+r_{a}\right)>\left(1+r_{2}\right)\left(1+r_{3}\right)$. Moreover, for any prices (by lemma 5 and 6$)$ and in any

7 For a $\beta-\delta$ we mean the implied date 1 preferences. 
optimal choice we must have $k_{3}=0$. As a result, the solution to the second period self must yield $c_{2}^{\star}\left(k_{2}, a\right)=w_{2}+\pi_{2}+k_{2}\left(1+r_{2}\right)$ and $c_{3}^{\star}\left(k_{2}, a\right)=w_{3}+\pi_{3}+$ $a\left(1+r_{a}\right)$. Hence, ${ }^{8} \frac{\partial c_{2}^{*}\left(k_{2}, a\right)}{\partial a}=0$ and $\frac{\partial c_{3}^{*}\left(k_{2}, a\right)}{\partial a}=1+r_{a}$ and similarly $\frac{\partial c_{2}^{*}\left(k_{2}, a\right)}{\partial k_{2}}=1+r_{2}$ and $\frac{\partial c_{3}^{*}\left(k_{2}, a\right)}{\partial k_{2}}=0$. Moreover, the market clearing conditions imply $c_{2}=f\left(a+k_{2}\right)+k_{2}$ and $c_{3}=f(a)+a$ together with factor prices $1+r_{2}=1+\mu_{2}+f^{\prime}\left(a+k_{2}\right), 1+r_{3}=1+$ $\mu_{3}+f^{\prime}(a)$ and $1+r_{a}=1+f^{\prime}(a)+\left(1-\mu_{3}+f^{\prime}(a)\right) f^{\prime}\left(a+k_{2}\right)$. The equilibrium allocation is determined by the two conditions following from the first order conditions for the consumer maximization (5):

$$
\begin{aligned}
& -u^{\prime}\left(c_{1}\right)+\beta \delta u^{\prime}\left(c_{2}^{\star}\left(k_{2}, a\right)\right) \frac{\partial c_{2}^{\star}\left(k_{2}, a\right)}{\partial a}+\beta \delta^{2} u^{\prime}\left(c_{3}^{\star}\left(k_{2}, a\right)\right) \frac{\partial c_{3}^{\star}\left(k_{2}, a\right)}{\partial a}=0, \\
& -u^{\prime}\left(c_{1}\right)+\beta \delta u^{\prime}\left(c_{2}^{\star}\left(k_{2}, a\right)\right) \frac{\partial c_{2}^{\star}\left(k_{2}, a\right)}{\partial k_{2}}+\beta \delta^{2} u^{\prime}\left(c_{3}^{\star}\left(k_{2}, a\right)\right) \frac{\partial c_{3}^{\star}\left(k_{2}, a\right)}{\partial k_{2}} \leq 0 .
\end{aligned}
$$

Substituting and denoting

$$
\begin{aligned}
\zeta_{1}\left(k_{2}, a, \mu_{3}\right):= & -u^{\prime}\left(f\left(k_{1}\right)+k_{1}-a-k_{2}\right)+\beta \delta^{2}\left(1+f^{\prime}(a)+\left(1+\mu_{3}+f^{\prime}(a)\right) f^{\prime}\left(a+k_{2}\right)\right) \\
& u^{\prime}(f(a)+a), \zeta_{2}\left(k_{2}, a, \mu_{2}\right):=-u^{\prime}\left(f\left(k_{1}\right)+k_{1}-a-k_{2}\right) \\
& +\beta \delta\left(1+\mu_{2}+f^{\prime}\left(a+k_{2}\right)\right) u^{\prime}\left(f\left(a+k_{2}\right)+k_{2}\right),
\end{aligned}
$$

the conditions for equilibrium allocations yield: $\zeta_{1}\left(k_{2}, a, \mu_{3}\right)=0$ and $\zeta_{2}\left(k_{2}, a, \mu_{2}\right) \leq 0$ with equality, if $k_{2}>0$.

Observe that $\zeta_{1}$ is continuous and strictly decreasing with $a$ and $k_{2}$ and strictly increasing with $\mu_{3}$. As a result the equation $\zeta_{1}\left(k_{2}, a^{\star}\left(k_{2}, \mu_{3}\right), \mu_{3}\right)=0$ determines a continuous function $a^{\star}$, that is strictly decreasing with $k_{2}$ and strictly increasing with $\mu_{3}$. Moreover, substituting $z:=a+k_{2}$ we have that the function $\tilde{\zeta}_{1}$ is strictly decreasing with $z$ and strictly increasing with $k_{2}$, where $\tilde{\zeta}_{1}\left(k_{2}, z, \mu_{3}\right)=-u^{\prime}\left(f\left(k_{1}\right)+k_{1}-z\right)+\beta \delta^{2}\left(1+f^{\prime}\left(z-k_{2}\right)+\left(1+\mu_{3}+f^{\prime}\left(z-k_{2}\right)\right)\right.$ $\left.f^{\prime}(z)\right) u^{\prime}\left(f\left(z-k_{2}\right)+z-k_{2}\right)$. Hence, although $a^{\star}$ is strictly decreasing with $k_{2}$, $k_{2} \rightarrow k_{2}+a^{\star}\left(k_{2}, \mu_{3}\right)$ is strictly increasing with $k_{2}$. Observe that $a^{\star}\left(k_{2}, \mu_{3}\right) \in$ $\left[0, f\left(k_{1}\right)+k_{1}-k_{2}\right]$. Next, consider $k_{2} \rightarrow \zeta_{2}\left(k_{2}, a^{\star}\left(k_{2}, \mu_{3}\right), \mu_{2}\right)$ and observe that

8 Clearly these partial derivatives are defined for interior points of domain (constrained date 2 consumer). If the date 2 consumer is not constrained, the one-sided derivative at the constraint point is equal to the one specified here, but not on the other side. However, as we already know the value of the utility cannot increase in this direction, i. e. $k_{3}$ must be zero. Hence, we can restrict our analysis to the case considered here. 
this is continuous and strictly decreasing. Finally, $\lim _{k_{2} \rightarrow f\left(k_{1}\right)+k_{1}} \zeta_{2}\left(k_{2}\right.$, $\left.a^{*}\left(k_{2}, \mu_{3}\right), \mu_{2}\right)=-\infty$. Hence, if $\zeta_{2}\left(0, a^{*}\left(0, \mu_{3}\right), \mu_{2}\right) \geq 0$, then we obtain the unique $k_{2}^{\star}\left(\mu_{2}, \mu_{3}\right)>0$ but if $\zeta_{2}\left(0, a^{\star}\left(0, \mu_{3}\right), \mu_{2}\right)<0$, we set $k_{2}^{\star}\left(\mu_{2}, \mu_{3}\right)=0$. In both cases we obtain the unique $a^{\star}\left(k_{2}^{*}\left(\mu_{2}, \mu_{3}\right), \mu_{3}\right)$.

We next consider the function $\Phi$, that fixed points are competitive equilibrium prices $r_{2}, r_{3}, r_{a}$ (normalized to consumption good price). For this reason, we let the price of the consumption good be denoted by $p$ and consider a simplex $\Delta \subset \mathbb{R}^{4}$, where for a vector $\left(r_{2}, r_{3}, r_{4}, p\right) \in \Delta$ its elements sum up to one. So take any $\left(r_{2}, r_{3}, r_{4}, p\right) \in \Delta$ and for $\frac{r_{2}}{p}, \frac{r_{3}}{p}, \frac{r_{a}}{p}$ consider the firm's maximization problem (1) with fixed $l_{t}=1, k_{1}, r_{1}=f^{\prime}\left(k_{1}\right)$. Then, maximization problem (1) is strictly concave with linearly independent constraints. Hence, there exists the unique Lagrange multiplier $\mu_{2}^{\prime}=\mu_{2}\left(\frac{r_{2}}{p}, \frac{r_{3}}{p}, \frac{r_{a}}{p}\right)$ and $\mu_{3}^{\prime}=\mu_{3}\left(\frac{r_{2}}{p}, \frac{r_{3}}{p}, \frac{r_{a}}{p}\right)$ that (from Berge maximum theorem) is continuous with parameters $\frac{r_{2}}{p}, \frac{r_{3}}{p}, \frac{r_{a}}{p}$. Next for such Lagrange multipliers $\mu_{2}^{\prime}, \mu_{3}^{\prime}$ solve $\zeta_{1}\left(k_{2}, a, \mu_{3}^{\prime}\right)=0$ and $\zeta_{2}\left(k_{2}, a, \mu_{2}^{\prime}\right) \leq 0$ as above to determine the unique $k_{2}^{*}\left(\mu_{2}^{\prime}, \mu_{3}^{\prime}\right)$ and unique $a^{*}\left(k_{2}^{*}\left(\mu_{2}^{\prime}, \mu_{3}^{\prime}\right), \mu_{3}^{\prime}\right)$, both continuous with $\mu_{2}^{\prime}, \mu_{3}^{\prime}$ and hence $r_{2}, r_{3}, r_{\mathrm{a}}$. In the final step update prices $r_{2}^{\prime}=\mu_{2}^{\prime}+f^{\prime}\left(a^{*}\left(k_{2}^{*}\left(\mu_{2}^{\prime}, \mu_{3}^{\prime}\right), \mu_{3}^{\prime}\right)+k_{2}^{*}\left(\mu_{2}^{\prime}, \mu_{3}^{\prime}\right), 1\right)$ and $r_{3}^{\prime}=\mu_{3}^{\prime}+f^{\prime}\left(a^{*}\left(k_{2}^{*}\left(\mu_{2}^{\prime}, \mu_{3}^{\prime}\right)\right.\right.$, $\left.\left.\mu_{3}^{\prime}\right)\right)$ and $r_{a}^{\prime}=f^{\prime}\left(a^{\star}\left(k_{2}^{\star}\left(\mu_{2}^{\prime}, \mu_{3}^{\prime}\right), \mu_{3}^{\prime}\right)\right)+\left(1+\mu_{3}^{\prime}+f^{\prime}\left(a^{\star}\left(k_{2}^{\star}\left(\mu_{2}^{\prime}, \mu_{3}^{\prime}\right), \mu_{3}^{\prime}\right)\right)\right) f^{\prime}\left(a^{\star}\left(k_{2}^{\star}\right.\right.$ $\left.\left.\left(\mu_{2}^{\prime}, \mu_{3}^{\prime}\right), \mu_{3}^{\prime}\right)\right)+k_{2}^{\star}\left(\mu_{2}^{\prime}, \mu_{3}^{\prime}\right)$. Observe that $\left(1+r_{a}^{\prime}\right) \leq\left(1+r_{2}^{\prime}\right)\left(1+r_{3}^{\prime}\right)$.

Finally normalize prices so that together with the price of the consumption good (i. e. $\frac{1}{r_{2}^{\prime}+r_{3}^{\prime}+r_{a+1}^{\prime}}$ ) they sum up to one, i. e. $\frac{r_{2}^{\prime}}{r_{2}^{\prime}+r_{3}^{\prime}+r_{a}^{\prime}+1}, \frac{r_{3}^{\prime}}{r_{2}^{\prime}+r_{3}^{\prime}+r_{a}^{\prime}+1}, \frac{r_{a}^{\prime}}{r_{2}^{\prime}+r_{3}^{\prime}+r_{a}^{\prime}+1}$ Next, define $\Phi: \Delta \rightarrow \Delta$ and observe that, by the above reasoning, it is a single valued and continuous, while $\Delta$ is a compact and convex set. By Brouwer fixed poind theorem there exists a fixed point of $\Phi$, say $\left(r_{2}^{*}, r_{3}^{*}, r_{a}^{*}, p^{*}\right)$. Taking that prices as given firm maximizes profits choosing the same allocation as the consumer. Observe that by construction $\left(1+r_{a}^{\star}\right) \leq\left(1+r_{2}^{\star}\right)\left(1+r_{3}^{\star}\right)$ and moreover $r_{2}^{\star}>0, r_{3}^{\star}>0, r_{a}^{\star}>0$. Hence, by lemma 5 and 6 we have that $u^{\prime}\left(c_{2}\right) \geq \beta \delta\left(1+r_{2}\right) u^{\prime}\left(c_{3}\right)$, unless $a^{*}=0$ but that cannot clear the market. This implies equilibrium existence.

Similar arguments can be used to verify equilibrium existence in the GP model. Take any $r=\left(r_{2}, r_{3}, r_{4}, p\right) \in \Delta$ and for $\frac{r_{2}}{p}, \frac{r_{3}}{p}, \frac{r_{a}}{p}$ consider the firm's maximization problem for given $l_{t}=1$ and $f^{\prime}\left(k_{1}\right)=r_{1}$. It is strictly concave with linearly independent constraints hence there exists the single valued Lagrange multiplier $\mu_{2}(r)$ and $\mu_{3}(r)$ continuous with $r$. Next consider the consumer's maximization problem. By lemma 2 and lemma 4 for any prices $\frac{r_{2}}{p}, \frac{r_{3}}{p}, \frac{r_{a}}{p}$ we must have $k_{2} k_{3}=0$. Hence we have two cases (i) $k_{2} \geq 0, k_{3}=0$ and (ii) $k_{2}=0, k_{3} \geq 0$. Start with (i) and write the first order conditions for the optimal choices of $a$ and $k_{2}$ (where we have already incorporated the market clearing conditions): $\zeta_{2}\left(k_{2}, a, r\right)=0$ and $\zeta_{2}\left(k_{2}, a, r\right) \leq 0$, where 


$$
\zeta_{1}\left(k_{2}, a, r\right):=-u^{\prime}\left(f\left(k_{1}\right)+k_{1}-a-k_{2}\right)-v^{\prime}\left(f\left(k_{1}\right)+k_{1}-a-k_{2}\right)+\beta_{2}\left(1+\frac{r_{a}}{p}\right) u^{\prime}(f(a)+a),
$$

and

$$
\begin{aligned}
\zeta_{2}\left(k_{2}, a, r\right):= & -u^{\prime}\left(f\left(k_{1}\right)+k_{1}-a-k_{2}\right)-v^{\prime}\left(f\left(k_{1}\right)+k_{1}-a-k_{2}\right) \\
& +\beta\left(1+\frac{r_{2}}{p}\right) u^{\prime}\left(f\left(a+k_{2}\right)+k_{2}\right) .
\end{aligned}
$$

Observe that $\zeta_{1}$ and $\zeta_{2}$ are continuous, strictly decreasing in $a$ and $k_{2}$. Hence there exists the unique $a^{\star}\left(k_{2}, r\right) \in\left[0, f\left(k_{1}\right)+k_{1}-k_{2}\right]$ such that $\zeta_{1}\left(k_{2}, a^{\star}\right.$ $\left.\left(k_{2}, r\right), r\right)=0$. Observe that $a^{\star}$ is continuous and strictly decreasing in $k_{2}$ but such that $k_{2} \rightarrow k_{2}+a^{\star}\left(k_{2}, r\right)$ is strictly increasing. As a result $k_{2} \rightarrow \zeta_{2}\left(k_{2}, a^{\star}\left(k_{2}, r\right), r\right)$ is continuous and strictly decreasing. Note that $\lim _{k_{2} \rightarrow f\left(k_{1}\right)+k_{1}} \zeta_{2}\left(k_{2}, a^{\star}\left(k_{2}, r\right), r\right)=-\infty$, hence either there exists the unique, continuous $k_{2}^{\star}(r)$ such that $\zeta_{2}\left(k_{2}^{\star}(r), a^{\star}\left(k_{2}^{\star}(r), r\right), r\right)=0$ or if $\zeta_{2}\left(0, a^{\star}(0, r), r\right) \leq 0$, then we set $k_{2}^{\star}(r)=0$. Now, verify if indeed $\zeta_{3}\left(a^{\star}\left(k_{2}^{\star}(r), r\right), k_{2}^{\star}(r), r\right) \leq 0$, where

$$
\zeta_{3}\left(k_{2}, a, r\right):=-u^{\prime}\left(f\left(a+k_{2}\right)+k_{2}\right)-v^{\prime}\left(f\left(a+k_{2}\right)+k_{2}\right)+\beta\left(1+\frac{r_{3}}{p}\right) u^{\prime}(f(a)+a) .
$$

If yes proceed, if not we need to consider case (ii), i. e. (ii) $k_{2}=0, k_{3} \geq 0$. Similarly, we obtain the first order conditions: $\tilde{\zeta}_{1}\left(k_{3}, a, r\right)=0$ and $\tilde{\zeta}_{3}\left(k_{3}, a, r\right) \leq 0$, where $\tilde{\zeta}_{1}\left(k_{3}, a, r\right):=-u^{\prime}\left(f\left(k_{1}\right)+k_{1}-a\right)-v^{\prime}\left(f\left(k_{1}\right)+k_{1}-a\right)+\beta^{2}\left(1+\frac{r_{a}}{p}\right) u^{\prime}\left(f\left(a+k_{3}\right)+a+k_{3}\right)$, and

$$
\tilde{\zeta}_{3}\left(k_{3}, a, r\right):=-u^{\prime}\left(f(a)-k_{3}\right)-v^{\prime}\left(f(a)-k_{3}\right)+\beta\left(1+\frac{r_{3}}{p}\right) u^{\prime}\left(f\left(a+k_{3}\right)+a+k_{3}\right) .
$$

Again note, that both $\tilde{\zeta}_{1}, \tilde{\zeta}_{3}$ are continuous, $\tilde{\zeta}_{1}$ is strictly decreasing in $a$ and $k_{3}$ hence there exists the unique, continuous and decreasing $a^{\star}\left(k_{3}, r\right)$ such that $\tilde{\zeta}_{1}\left(k_{3}, a^{\star}\left(k_{3}, r\right), r\right)=0$. Observe also that $k_{3} \rightarrow k_{3}+a^{\star}\left(k_{3}, r\right)$ is strictly increasing. As a result $k_{3} \rightarrow \tilde{\zeta}_{3}\left(k_{3}, a^{\star}\left(k_{3}, r\right), r\right)$ is strictly decreasing. Again there exists the unique $k_{3}^{*}$ such that $\tilde{\zeta}_{3}\left(k_{3}^{*}(r), a^{*}\left(k_{3}^{*}(r), r\right), r\right)=0$ or we set $k_{3}^{*}(r)=0$. We finally confirm that $\tilde{\zeta}_{2}\left(k_{3}^{\star}(r), a^{\star}\left(k_{3}^{\star}(r), r\right), r\right) \leq 0$, where

$$
\begin{gathered}
\tilde{\zeta}_{2}\left(k_{3}, a, r\right):=-u^{\prime}\left(f\left(k_{1}\right)+k_{1}-a\right)-v^{\prime}\left(f\left(k_{1}\right)+k_{1}-a\right)+\beta\left(1+\frac{r_{2}}{p}\right) \\
{\left[u^{\prime}\left(f(a)-k_{3}\right)+v^{\prime}\left(f(a)-k_{3}\right)-v^{\prime}(f(a))\right] .}
\end{gathered}
$$

Clearly, this is either (i) or (ii), unless both $k_{2}^{\star}(r)=0$ and $k_{3}^{\star}(r)=0$. In the verified case we denote by $a^{\star}(r)$ the implied $a^{\star}\left(k_{2}^{\star}(r), r\right)$ or $a^{\star}\left(k_{3}^{\star}(r), r\right)$. As the (case) 
selection process ${ }^{9}$ is also continuous, we obtain the continuous supply functions $k_{2}^{\star}, k_{3}^{\star}, a^{\star}$.

Having them, we update prices: $r_{2}^{\prime}(r)=\mu_{2}(r)+f^{\prime}\left(a^{\star}(r)+k_{2}^{\star}(r)\right)$ and $r_{3}^{\prime}(r)=\mu_{3}(r)+f^{\prime}\left(a^{\star}(r)+k_{3}^{\star}(r)\right)$ and $\left.r_{a}^{\prime}(r)=f^{\prime}\left(a^{\star}(r)+k_{3}^{\star}(r)\right)\right)+\left(1+\mu_{3}(r)+f^{\prime}\left(a^{\star}(r)\right.\right.$ $\left.\left.+k_{3}^{\star}(r)\right)\right) f^{\prime}\left(a^{\star}(r)+k_{2}^{\star}(r)\right)$. Finally, normalize prices so that together with the price of the consumption good they sum up to one. ${ }^{10}$ Define a function $\Phi$ on $\Delta$ and by Brouwer fixed point theorem take its any fixed point. By construction, these are the equilibrium prices and are strictly positive. Indeed, at the fixed point the consumer maximization and market clearing imply prices, such that the firm's maximization conditions produce the same allocation, as determined by the consumer's first order conditions.

The reason we obtain equilibrium existence in our model is the presence of the commitment assets together with credit constraints and active firms. In fact, the corner allocation results we obtained in the previous lemmas are necessary to avoid problems mentioned by Gabrieli and Ghosal (2013) and presence of the firm helps avoid problems identified by Kocherlakota (2001). Finally, due to the corner allocation, the standard toolkit to show equilibrium existence via first order conditions does not work.

To sum up, the above propositions imply that in both models we observe positive quantity of a commitment (illiquid) asset and zero quantity of some of the short-lived (liquid) assets.

Although similar, the results of both models can be different. The difference is that in the GP model we can observe positive investment in the long term asset $\left(k_{3}>0\right)$ with $k_{2}=0$, while in the $\beta-\delta$ model we cannot. The reason comes from the fact that, although in both models there are few ways (depending on the level of $k_{2}$ ) to provide income to finance the same level of consumption $c_{2}$ and $c_{3}$, they cause various cost of self control in the GP model, while cause no effects in the $\beta-\delta$, until $c_{2}$ and $c_{3}$ do not change.

To see that, it is indeed possible, consider the following example. Let $u(c)=\ln c, v(c)=\alpha \ln c, F(k, 1)=A k^{\gamma}$. For parameters values: $A=5, \beta=0.9$, $\gamma=0.5, \alpha=0.5, m_{1}=2$ the unique solution is $a \approx 0.76, c_{1} \approx 1.24, c_{2} \approx 4.31$, $c_{3} \approx 4.49, r_{2} \approx 2.87+\mu_{2}, r_{3} \approx 2.78, r_{a} \approx 13.6$. For such solution, indeed, the firm uses (relatively cheep) asset $a$ and adjusts production using the cheaper

9 Indeed, observe that $k_{2}=0=k_{3}$ is the case continuously linking conditions in (i) and (ii).

10 For this step, demand must me homogeneous of degree zero. Clearly it is problematic, if preferences are price-dependent, e.g. via $-v\left(p\left(\pi_{2}+w_{2}+\left(1+r_{2}\right) \frac{k_{2}}{p}\right)\right)$ term. Clearly, it is not possible in our case, as this is either $v\left(c_{2}\right)=v\left(m_{2}\right)$ or $m_{2}=\pi_{2}+w_{2}$. 
from the short term capitals, namely $k_{3}$. Similarly, the consumer does not want to invest in $k_{2}$, as this increases the cost of self control and reduces (small) income available in date 1.

\section{Comments and Conclusion}

In this paper we have compared a general equilibrium of the two models: one, where households choices are determined in the GP model and the other, where households choices come from the $\beta-\delta$ model. We show that equilibrium allocations of both models (GP and $\beta-\delta$ ) imply positive consumption of the commitment asset and corner consumption of one of the short term assets. We also show that, when the production sector is incorporated into the economy, we can restore the equilibrium existence (without recalling measure space of consumers). These are two new results as compared to the literature (see Kocherlakota (2001), Gabrieli and Ghosal (2013) and Gul and Pesendorfer (2004b)). This also shows that the symmetric equilibrium non-existence in Kocherlakota (2001) results from the assumption of fixed supply of assets. Few other comments follow.

1. The composition of both short term assets (bonds) can be treated as a long term (liquid) asset that pays $\left(1+r_{2}\right)\left(1+r_{3}\right)$ by non-arbitrage condition. We now formally show, that indeed the non-arbitrage condition is satisfied for markets with both types of tempted consumers. To see that, suppose we add the additional long-term (liquid) asset $\tilde{k}$, that pays $1+\tilde{r}$ in date 3 and denote its level in date 2 by $\tilde{k}_{2}$ and in date 3 by $\tilde{k}_{3}$.

For the sake of argument suppose that $(1+\tilde{r})>\left(1+r_{2}\right)\left(1+r_{3}\right)$. Then, we must have $\tilde{k}=0$ solving the firm's maximization problem. Indeed, if it is not true the firm could increase profits by replacing $\tilde{k}$ by $k_{2}$ and $k_{3}$ so that to keep the level of production unchanged and generating additional profits from cheaper rent of capital. However, for such relation of prices the consumer will always require positive amount of $\tilde{k}$ contradicting market clearing. The last argument is delicate as it requires analysis of temptations. We do it for both types of consumers separately.

We start with the GP consumer. As $\tilde{k}$ is liquid, it changes date 2 temptation and the consumer's problem becomes:

$$
\begin{aligned}
\max _{k_{2}, k_{3}, \tilde{k}_{2}, \tilde{k}_{3}, a \geq 0} & \sum_{t=1}^{2} \beta^{t-1}\left\{u\left(c_{t}\right)+v\left(c_{t}\right)\right\}+\beta^{2} u\left(c_{3}\right)-\beta v\left(w_{2}+\pi_{2}+k_{2}\left(1+r_{2}\right)+\tilde{k}_{2} \frac{(1+\tilde{r})}{1+r_{3}}\right), \\
& c_{1}+k_{2}+\tilde{k}_{2}+a=w_{1}+\pi_{1}+k_{1}\left(1+r_{1}\right), \\
& c_{2}+k_{3}+\tilde{k}_{3}=w_{2}+\pi_{2}+k_{2}\left(1+r_{2}\right)+\tilde{k}_{2} \frac{(1+\tilde{r})}{1+r_{3}}, \\
& c_{3}=w_{3}+\pi_{3}+k_{3}\left(1+r_{3}\right)+\tilde{k}_{3}(1+\tilde{r})+a\left(1+r_{a}\right) .
\end{aligned}
$$


For the sake of argument suppose $\tilde{k}_{2}=\tilde{k}_{3}=0, k_{2}>0$ and $k_{3}>0$. We show this cannot be the optimal choice. So consider a modified choice, where we reduce $k_{2}$ by $\varepsilon$ and $k_{3}$ by $\left(1+r_{2}\right) \epsilon$. We compensate this change with the increase in $\tilde{k}_{2}$ by $\frac{\left(1+r_{2}\right)\left(1+r_{3}\right)}{(1+\tilde{r})} \epsilon$ and $\tilde{k}_{3}$ by $\left(1+r_{2}\right) \epsilon$. Observe that this change leaves the temptation level unchanged (as $m_{2}$ is constant, while it frees up some resources in date 1 and 3 allowing to increase $c_{1}$ and $c_{2}$. Similar consideration goes through, if only one of $k_{t}$ is positive. Hence, we can propose a change, that leaves the temptation unaffected and frees up some resources in date 1 or 2 . We can similarly proceed for $(1+\tilde{r})<\left(1+r_{2}\right)\left(1+r_{3}\right)$.

Now consider the $\beta-\delta$ consumer. Again suppose $\tilde{k}_{2}=\tilde{k}_{3}=0, k_{2}>0$ and $k_{3}>0$. The same modification as proposed above leaves $m_{2}$ unchanged and generates extra resources in date 1 and 3. The same argument holds for the corner cases.

As a result, we argue that in both models we must have $(1+\tilde{r})=\left(1+r_{2}\right)\left(1+r_{3}\right)$ or perhaps that $\tilde{k}_{2}=\tilde{k}_{3}=k_{2}=k_{3}=0$. This implies that the term structure of the (liquid) bond prices cannot reveal the time consistency/self control problems and typical non-arbitrage condition hold. Again the only way to test for consumers' time-consistency or their self control problems is to compare the returns or prices of commitment (illiquid) assets versus liquid ones, but such that can be used to finance the same consumption in the future periods.

2. Our analysis shows similarities and differences in the demand and supply of assets for a three-period economy with GP or $\beta-\delta$ consumers. It is interesting to see, whether the similarities and corner allocation results are still valid for $T$-period economy. To build an intuition behind results for both types of preferences, we consider $T=4$ period economy with short (one period) assets $\left\{k_{t}\right\}_{t=2}^{4}$ and two longterm (illiquid) commitment assets $a_{1}^{3}, a_{1}^{4}$ purchased in date 1 and giving a return of $\left(1+r_{a_{1}^{3}}\right)$ and $\left(1+r_{a_{1}^{4}}\right)$ in date 3 and 4 respectively. Such assets allow to finance consumption in period 3 and 4 with controlled temptations in period 2 and 3.

For the economy with GP preferences, similarly as above, we claim that for $\left(1+r_{a_{1}^{4}}\right) \geq\left(1+r_{a_{1}^{3}}\right)\left(1+r_{4}\right) \geq\left(1+r_{2}\right)\left(1+r_{3}\right)\left(1+r_{4}\right)$, we have $a_{1}^{3} k_{4}=0, k_{2} k_{3} k_{4}=0$ and $k_{2} k_{3}=0$. Analysing optimal choices of firms, we conclude that in equilibrium we must observe:

$$
\left(1+r_{a_{1}^{4}}\right) \leq\left(1+r_{a_{1}^{3}}\right)\left(1+r_{4}\right) \leq\left(1+r_{2}\right)\left(1+r_{3}\right)\left(1+r_{4}\right),
$$

and Inada assumption on $\mathrm{u}$ implies that in equilibrium $a_{1}^{4}>0$ and that some of the short term assets must be zero.

For the same assets consider the $\beta-\delta$ consumer. Similarly as above we claim that in equilibrium:

$$
\left(1+r_{a_{1}^{4}}\right) \leq\left(1+r_{a_{1}^{3}}\right)\left(1+r_{4}\right) \leq\left(1+r_{2}\right)\left(1+r_{3}\right)\left(1+r_{4}\right),
$$


which, by Inada conditions on $\mathrm{u}$, implies that $a_{1}^{4}>0$ and $k_{4}=0$. Moreover $k_{3}=0$ or $k_{2}=a_{1}^{3}=0$.

Again, the two models similarly imply that one of the long term commitment assets is in the positive supply and some of the short term assets are not traded in equilibrium. There are differences, however. For the $\beta-\delta$ model we have more implications (corner solutions) to test, than in the GP model.

Clearly in the economy with many periods one can consider different structures of commitment assets. For example, if $T=4$ we could alternatively consider $a_{1}^{3}, a_{2}^{4}$ but then equilibrium characterization of prices and allocations would be different. The intuition here is that in order to finance consumption in period 4 using the illiquid asset one needs to either transfer some liquid asset to period 2 or invest in the illiquid asset $a_{1}^{3}$ and then transfer some liquidity to period 4 using liquid $k_{4}$. Both ways involve some loss of efficiency due to temptation costs. Hence, the possibility of obtaining corner equilibria in such model is limited. Nevertheless, the analysis presented in this paper can be easily extended to cover such cases as well.

3. The Gul and Pesendorfer (2004a) model of a (dynamic) choice with temptations is not the only specification of temptation we can consider. Various extensions of this basic approach can be analyzed, including random temptations (see Dekel, Lipman, and Rustichini (2009)), dynamic temptations (see Noor (2007)) or choice dependent temptation cost (see Olszewski (2011)), bringing some new insights to the analysis of asset prices under temptations. Similarly, for the time-inconsistency model. The quasi-hyperbolic discounting model is not the only one that we can consider. More general hyperbolic discount factors or time-changing preferences and various consumer's behaviour (from naive to sophisticated) implications on asset prices are worth analyzing (see Gabrieli and Ghosal (2013) or Herings and Rohde (2006)).

Acknowledgements: I want to thank Paweł Dziewulski, Piotr Maćkowiak, Kevin Reffett and two anonymous referees for some useful comments during the writing of this paper.

Funding: Financial support from the NCN grant DEC-2013/11/D/HS4/03813 is greatly acknowledged.

\section{References}

Ameriks, J., A. Caplin, J. Leahy, and T. Tyler. 2007. “Measuring Self-Control Problems.”

American Economic Review 97:966-72. 
Angeletos, G.-M., D. Laibson, A. Repetto, J. Tobacman, and S. Weinberg. 2001. "The Hyberbolic Consumption Model: Calibration, Simulation, and Empirical Evaluation.” Journal of Economic Perspectives 15:47-68.

Balbus, Ł., K. Reffett, and Ł. Woźny. 2015. "Time Consistent Markov Policies in Dynamic Economies with Quasi-Hyperbolic Consumers." International Journal of Game Theory 44:83-112.

Dekel, E., B. L. Lipman, and A. Rustichini. 2009. "Temptation-Driven Preferences." Review of Economic Studies 76:937-71.

Dziewulski, P. 2015. "Efficiency of Competitive Equilibria in Economies with Time-Dependent Preferences." Journal of Economic Theory 159:311-25.

Gabrieli, T., and S. Ghosal. 2013. "Non-Existence of Competitive Equilibria with Dynamically Inconsistent Preferences." Economic Theory 52:299-313.

Gul, F., and W. Pesendorfer. 2001. "Temptation and Self-Control." Econometrica 69:1403-35.

Gul, F., and W. Pesendorfer. 2004a. "Self-Control and the Theory of Consumption." Econometrica 72:119-58.

Gul, F., and W. Pesendorfer. 2004b. "Self Control, Revealed Preferences and Consumption Choice." Review of Economic Studies 7:243-64.

Herings, P., and K. Rohde. 2006. "Time-Inconsistent Preferences in a General Equilibrium Model." Economic Theory 29:591-619.

Kocherlakota, N. R. 2001. "Looking for Evidence of Time-Inconsistent Preferences in Asset Market Data.” Quarterly Review 25:13-24.

Krusell, P., B. Kuruş̧̧u, and A. A. J. Smith. 2002. "Equilibrium Welfare and Government Policy with Quasi-Geometric Discounting." Journal of Economic Theory 105:42-72.

Krusell, P., B. Kuruscu, and A. A. J. Smith. 2010. "Temptation and Taxation.” Econometrica 78:2063-84.

Laibson, D. 1997. “Golden Eggs and Hyperbolic Discounting." Quarterly Journal of Economics 112:443-77.

Luttmer, E. G. J., and T. Mariotti. 2003. "Subjective Discounting in an Exchange Economy." Journal of Political Economy 111:959-89.

Luttmer, E. G. J., and T. Mariotti. 2006. "Competitive Equilibrium When Preferences Change Over Time.” Economic Theory 27:679-90.

Luttmer, E. G. J., and T. Mariotti. 2007. "Efficiency and Equilibrium When Preferences are TimeInconsistent." Journal of Economic Theory 132:493-506.

Noor, J. 2007. "Commitment and Self-Control." Journal of Economic Theory 135: 1-34.

Olszewski, W. 2011. "A Model of Consumption-Dependent Temptation." Theory and Decisions 70:83-93.

Rogerson, R. 1988. “Indivisible Labor, Lotteries and Equilibrium.” Journal of Monetary Economics 21:3-16.

Strotz, R. H. 1956. "Myopia and Inconsistency in Dynamic Utility Maximization." Review of Economic Studies 23:165-80.

Woźny, Ł. 2015. "On Incentives, Temptation and Self-Control." Mathematical Social Sciences 74:60-7.

Yilmaz, M. 2013. “Repeated Moral Hazard with a Time-Inconsistent Agent.” Journal of Economic Behavior and Organization 95:70-89. 